\title{
Research on streaming media adaptive transmission control strategy
}

\author{
Yunxiang Zhang ${ }^{1, a}$,Yunpeng $\mathrm{He}^{2, b}$, Chuanhui $\mathrm{Li}^{3, \mathrm{c}}$ and Jinxia Huang ${ }^{4, \mathrm{~d}}$ * \\ ${ }^{1}$ Business College Jiamusi University, Jiamusi China \\ ${ }^{2}$ Radio and Television Bureau of Jiamusi , iamusi China \\ ${ }^{3}$ Business College Jiamusi University,Jiamusi China \\ ${ }^{4}$ College of Information Science \& Electronic Technology,Jiamusi University,Jiamusi China

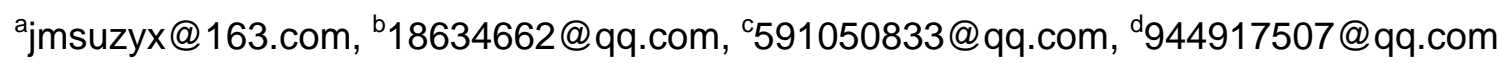

Keywords: streaming media; adaptive; control strategy

\begin{abstract}
This paper first introduces the basic principle of the streaming media adaptive transmission, explains the flow of two inseparable part media adaptive transmission technique in adaptive coding: streaming media and streaming media adaptive transmission control strategy, and analyzes the current several kinds of streaming media transmission control strategy of adaptive advantages and disadvantages, presents a new streaming media adaptive strategy. To put forward a comprehensive quality obtained above strategy better streaming adaptive transmission strategy and provide a theoretical basis.
\end{abstract}

\section{Adaptive streaming media transmission principle}

With the rapid development of network communication, network bandwidth than has been previously have been significantly improved, real-time audio, video streaming transmission in the Internet online may be. Considering that most of the streaming media applications, users of streaming media transmission in real-time demand is far greater than the reliability, therefore, the system shall be able to timely evaluate the state of the network, dynamically adjust the coding end sent to code traffic on the network, to make full use of network bandwidth as much as possible to avoid the big delay or congestion, ensure that the transmission of streaming media best the quality of service.

The basic idea of adaptive transmission is the sending end according to the feedback information to predict the status of the network side of the receiver, and accordingly adopts some strategies to realize the dynamic adjustment of the sending rate, make it adapt to changes in network bandwidth. Due to the time-varying channel, the use of adaptive transmission scheme can prevent the full use of channel capacity to happen. Random burst loss and excessive delay, is devastating to the video quality, using adaptive transmission control strategy is excellent, can make full use of network bandwidth, improve the efficiency of transmission of video data, ensure that the transmission of streaming media service quality.

Streaming media adaptive transmission consists of two inseparable parts: adaptive code stream media and stream media adaptive transmission control strategy.

Adaptive rate control module through the received RTCP packet network bandwidth estimation of current, then using the adaptive transmission strategy effective, adaptive coding of audio and video, so that the audio and video rate and network bandwidth, can be used to avoid network congestion.

Audio and video coding technology of various popular at present, such as MPEG, H.26X are adaptive, can provide the implementation complexity scalability in time, space and audio and video compression. Adaptive transmission control technology to dynamic detection network bandwidth changes, using the transmission control strategy effectively make the audio and video transmission rate and network bandwidth adaptation, adaptive audio and video coding technology and adaptive transmission control strategy combining can flexibly adapt to the change of network bandwidth, provide continuous and stable audio, video quality. 


\section{Adaptive code stream media}

Adaptive coding for the purpose of obtaining the best coding quality at a given coding rate case, through the quantization parameter adjustment or adjust the frame rate to achieve various code.

\section{To adjust the frame rate}

Frame rate is high, i.e. the image frames per second delivered more, better video quality. Adjust the frame rate can be achieved by selecting frame technology. Choose the frame technology refers to the video stream according to certain rules of recombinant video frame, and then according to the network bandwidth estimation system, dynamic adjustment of sending the frame rate, which adapts to the network transmission.

To adjust the frame rate is simple to realize, and has the following advantages: first, at the sending end to have the option of discarding some data frame, reduces the network congestion may occur; second, the transmitted data frame sending end using intelligent methods have a choice, improves the data to be transmitted on the usability of the receiver, which improves the overall the quality of video transmission.

Adjust the quantization parameter

Adjust the frame rate is to achieve adaptive in the audio and video encoded by selectively discarding some frames, and adjust the quantization parameter is the audio and video coding quantization parameter setting code before coding, is relatively complex.

\section{Streaming media adaptive transmission strategy}

\section{Factors to be considered in adaptive transmission strategy}

(1) TCP friendliness

When the TCP flows and UDP flows share the network bandwidth, UDP flow will be further occupation of network bandwidth, making the TCP flow and UDP is not fairness between flows. Therefore, the application of streaming media should be adaptive transmission strategy with TCP friendliness. TCP friendliness is the meaning of TCP flow and a self adaptive flow such as UDP flow through the same path similarity propagation characteristics, and have the same round trip time (RTT) and packet loss rate, should have similar bandwidth.

(2) convergence

Due to the random change of network state, rate of streaming media transmission must also be changed at any time. Convergence is usually used from the initial state of congestion the desired steady state time or speed to measure. Streaming media adaptive transmission strategy should be good can rapidly converge.

(3) stability (smoothness)

Effective streaming adaptive transmission strategy of streaming media transmission quality effect is stable, can reduce a lot of streaming media transmission quality fluctuation.

\section{Analysis of stream media adaptive transmission strategy}

(1) adaptive transmission strategy based on source, including the rate control based probe method and model method based on rate control.

Based on the basic thought of the control rate of exploration are the sending end according to the network packet loss feedback rate, the current network available bandwidth is divided into free, full load and congestion in three states. When the network is idle, a linear increase in sending rate; when the network is congested, the multiplicative decrease the transmission rate; when the network is fully loaded, the sending rate constant. The specific implementation is as follows:

Firstly, according to the network packet loss rate, feedback through a low pass filter lambda $=(1$ alpha) + alpha beta (beta is the network packet loss rate, $0<$ alpha $<1$ ) loss rate calculation of smooth, can avoid excessive volatility and lambda will according to network state is divided into free, full load and congestion three state. As shown in figure.1: 


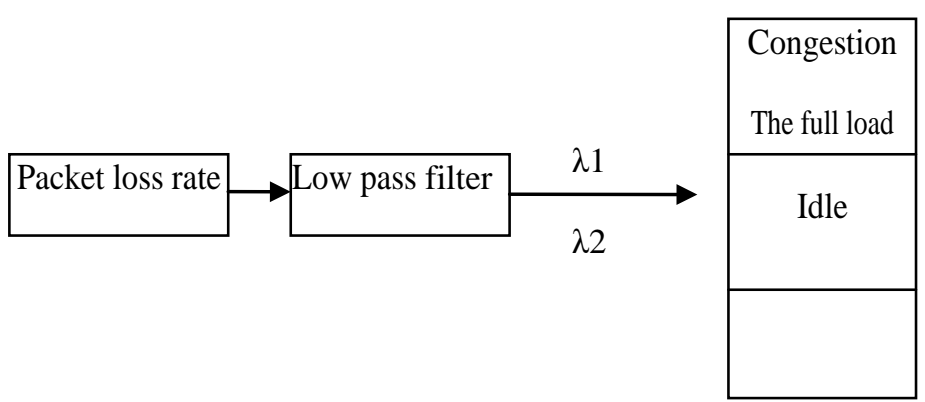

Fig.1: Classification of network state

Finally, according to the obtained network state, by using a kind of adaptive algorithm to adjust the sending rate.AIMD is the main adaptive algorithm used (additive increase multiplicative decrease) and PMIMD (proportional multiplicative increase and multiplicative decrease) algorithm. AIMD algorithm is as follows:

$\operatorname{If}(\lambda<\lambda 2) \quad S=\min (S+v, S \max )$

Else if $(\lambda>\lambda 1) \quad S=\max (S \times \mu, S \sin )$

Else $\mathrm{S}=\mathrm{S}$

Where lambda is low pass filtered through the packet loss rate, lambda 1 between full and congestion threshold, lambda 2 idle and full load between the threshold, $\mathrm{S}$ transmission rate, $\mathrm{Mu}$ is the multiplicative decrease factor, $\mathrm{V}$ factor for the increase of additive, Smax and Smin are respectively transmit maximum sending rate and the minimum hair send rate.

Rate of probe method control has the advantages of its working principle and stability, fairness has been based on the characteristics we are familiar with, the realization is very simple. But its defect is that the sending rate changes, the application flow of sudden strong, jitter, especially when the network condition by loaded into congestion.

Rate control and prospecting model method does not explicitly estimate the available bandwidth of network based on the formula of different throughput model, by using the method of a TCP connection calculation transmission rate, congestion that video connection to avoid the use of TCP like mode, realizing fair competition and TCP flow rate, so the model law control methods the rate based TCP friendly, and can explicitly estimate the available bandwidth of network. Throughput model formula is as follows:

$$
\mathrm{S}=1.22
$$

$\times \mathrm{MTU} /(\mathrm{RTT} \times \mathrm{P} 1 / 2)$

\section{(1)}

Where $\mathrm{S}$ is the transmission rate, MTU is the maximum packet size, value can be 576Byte RTT as the default, the sending end to the receiving end of round trip time, packet loss when transmitting $\mathrm{P}$ allowed rate.

(2) Adaptive transmission strategy based on the receiving end, the sender is not involved in the rate control, and by the receiver by reducing or increasing the number of channels for rate adjustment.

Adaptive transmission strategy of the receiving end of the need for layered coding of video based on [38]. Layered coding is to compress an original video sequence into a base layer and several enhancement layers, the basic layer can be independently decoded to provide lower video quality, the enhancement layer and basic layer together is decoded, provide higher quality video. The receiving end according to its network status receive different number of layers, receiving more layers, the better the quality of received video.

And adaptive transmission strategy based on source based on adaptive transmission strategy is similar, the receiving end is also divided into two categories: rate control based probe method and model method based on rate control.

\section{Streaming media adaptive transmission strategy}

AIMD and some other adaptive transmission strategy based on source, its disadvantages have been discussed in the front, is sending rate changes, the application flow of sudden strong, jitter, especially when the network condition by loaded into congestion. The reason is, the sending end according to the network packet loss feedback rate, the current network bandwidth is divided into 
free, full load and congestion in three states. When the network is idle, a linear increase in sending rate; when the network is congested, the multiplicative decrease the transmission rate; when the network is fully loaded, the sending rate constant. However, when network congestion, packet loss rate and packet delay will increase. Therefore, only rely on the existence of one-sided sex adjusted loss rate to the sending end output rate, will result in the increase of calculation error transmission rate. On the other hand, when the network condition by loaded into congestion, sending rate by method of reduced easily lead to rate volatility changes, thus affecting the quality of the video playback client. Here, put forward a new adaptive transmission strategy of streaming media.

(1) The introduction of a low pass filter $\lambda=(1-\alpha)+\alpha \beta(\beta$ is the network packet loss rate, $0<\alpha<1)$ calculated by smoothing the packet loss rate.

(2) If $\lambda<\lambda 2$ ( $\lambda 2$ network is in an idle and full of critical point), the additive increase transmission rate of $\mathrm{S}=\min (\mathrm{S}+\mathrm{V}, \mathrm{Smax})$, jump to the (1).

(3) If $\lambda>\lambda 1$ ( $\lambda 1$ network is in congestion and full of critical point), multiplicative decrease transmission rate, $S=\max (S \times \mu, S m i n) S$, jump to the (1).

(4) If $\lambda 1<\lambda<\lambda 2$, The calculated delay jitter in K networks.

(5) If $\mathrm{K}>0$, Indicate that the network is gradually turning to the congestion, adjusts the sending rate, $S=\min (S-v / 2, S m a x)$, jump to the $(1)$. (K for the delay jitter)

(6) If $\mathrm{K}<0$, The network gradually free, adjust the sending rate, $\mathrm{S}=\min \left(\mathrm{S}+2^{*} \mathrm{v}, \mathrm{Smax}\right)$, jump to the (1).

(7) If $\mathrm{J}=0$, Shows that the network did not change, the sending rate constant, $S=S$, jump to the (1).

From the above transmission strategy can be seen, the sending end according to the calculated output rate jitter of two kinds of factors of the network packet loss rate and network delay. Because the network packet loss rate reflects the current network status, and the network delay jitter indicates a period of time in the future. Network congestion may occur in the network, so the full, according to the change trend of network delay jitter and timely forecast network congestion in the future period of time, to fine tune the sending rate, can smooth the good streaming data flow, as far as possible to avoid congestion occurrence or make full use of network bandwidth, so as to achieve the optimum video transmission quality of service.

When the network delay is not very large, the adaptive transmission algorithm based on the sending end can provide better video transmission quality of service. When the network delay is very large, the receiving end in a period of time to receive the video data stream, the receiving end will appear the screen stuck situation. Aiming at this problem, at the receiving end is the realization of a buffer control algorithm:

(1) at a certain time $\Delta t$, obtains the current network delay jitter of K1;

(2) if the K1>0, then jump to the (3); otherwise, jump to the (1);

(3) in the next $\Delta t$, obtains the current network delay jitter of $\mathrm{K} 2$;

(4) if the $\mathrm{K} 2>\mathrm{K} 1$, then adjust the receiving buffer size of $\mathrm{M}=\min (\mathrm{M} \times \alpha, M \max )(\alpha$ is a coefficient, $1<\alpha<2$, Mmax is the maximum size of the buffer), jump to the (1);

(5) if the $\mathrm{K} 2<\mathrm{K} 1$, then adjust the receiving buffer size of $\mathrm{M}=\max (\mathrm{M} \times \beta, \mathrm{Mmin})$ ( $\beta$ is a coefficient, $0<\alpha<1$, Mmin is the minimum buffer size), jump to the (1);

Through the network congestion status forecast next period, increasing or reducing buffer receiving end. When the delay gradually increases, with large buffer to increase the receiving end video playback time, playback of the video stream while waiting for the arrival of new filling the buffer; when the delay gradually decreases, reducing the size of the buffer, in order to reduce the overhead of receiver system. 


\section{The simulation and analysis}

(1) To set up the simulation environment as shown in figure.2.

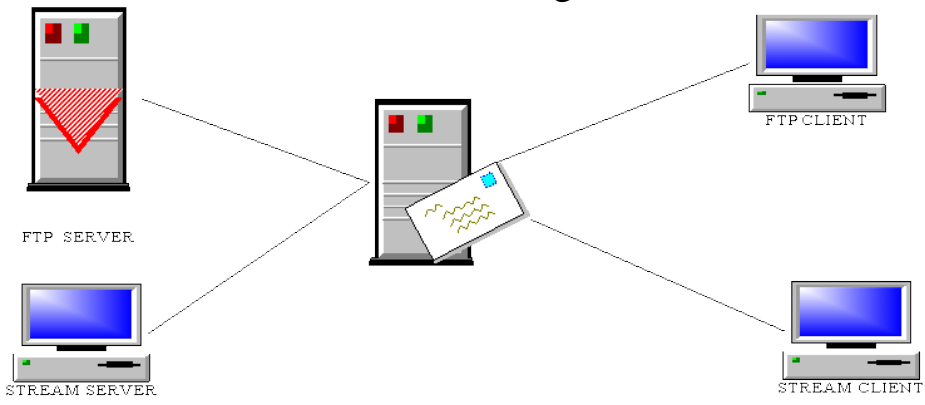

Fig. 2: IP network simulation topology

Experiment one: Transmission Simulation for FTP application and no transmission strategy of streaming media application, the application of FTP 0s from the start, duration of 25s, the application of streaming media 10s from the start, continuous 5S. After the simulation, respectively collecting link bandwidth throughput and packet loss number. The experimental results in Figure.3 and figure.4.

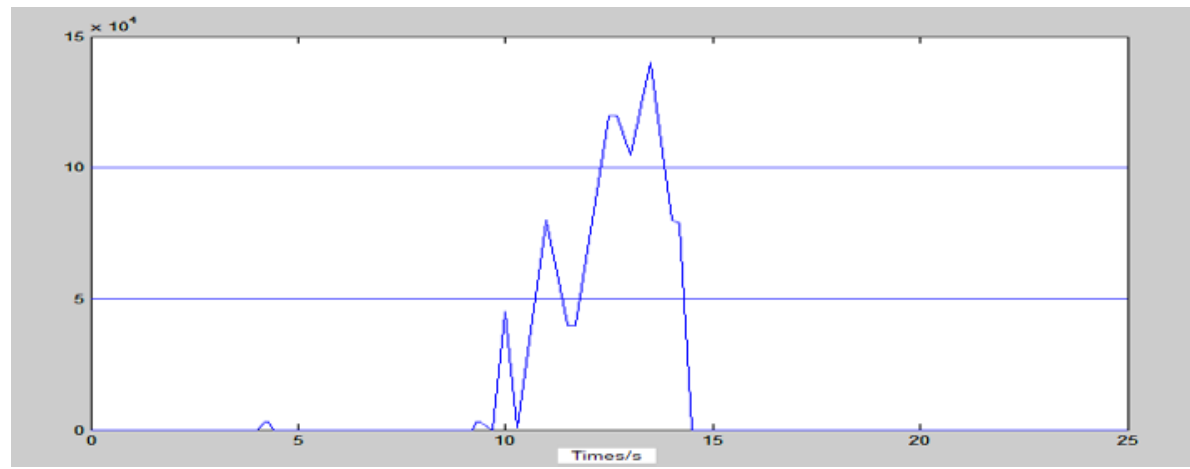

Fig. 3: The link bandwidth throughput no transmission strategy

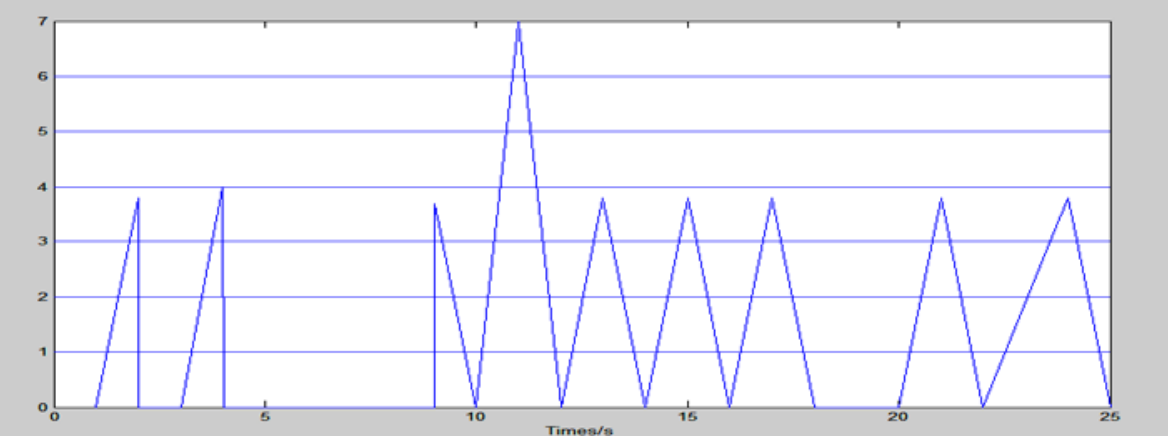

Fig. 4: Network packet loss free transmission strategy number

Experiment two: FTP application and transmission simulation application of streaming media transmission strategy, the application of FTP 0s from the start, duration of 25s, the application of streaming media 10s from the start, continuous 5S. After the simulation, respectively collecting link bandwidth throughput and packet loss number. The experimental results in Figure.5 and figure. 6.

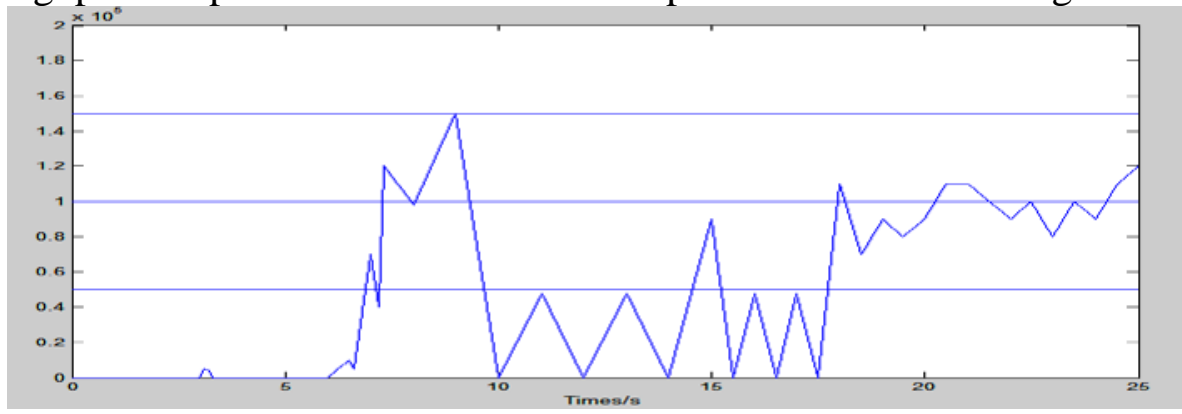

Fig.5: Improved link bandwidth throughput transmission strategy 


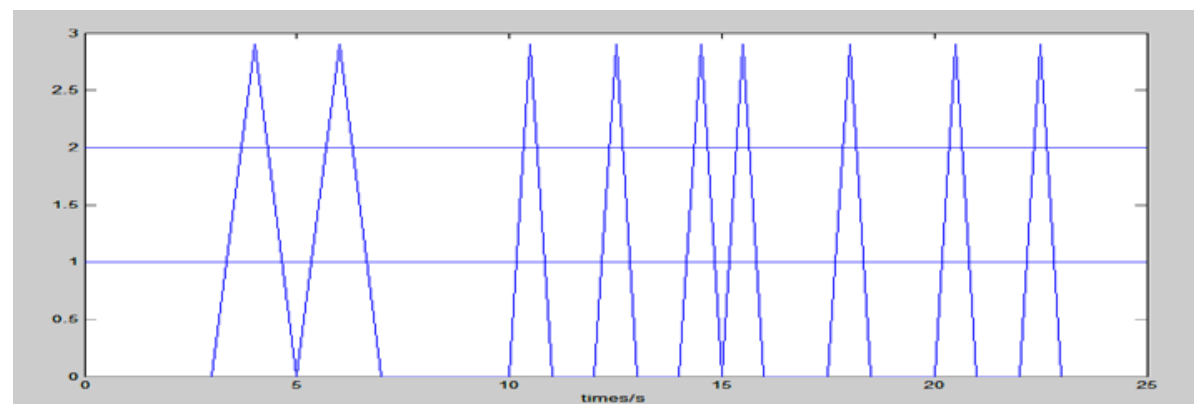

Fig. 6: Improving the network packet loss transmission strategy number

(3) the result analysis

Analysis of link bandwidth throughput: comparing Figure 3 and figure 5, in the aftermath of the 1s, the FTP application started to build, after 10s, the application of streaming media began to build, not using the simulation of adaptive transmission strategy, the FTP flow is reduced to 0, streaming media flow occupy a lot of bandwidth, so as to affect the transmission of other TCP applications, does not have the TCP has good; using adaptive transmission strategy, FTP flow has decreased, but still with the streaming media transmission flow together, common share network bandwidth. Through the comparative analysis, can explain the transmission strategy with TCP friendliness.

Analysis of network packet loss number: before 10s, the number of network packet loss figure 4 and Figure 6 are approximately the same, after 12s, without using the packet loss simulation of adaptive transmission strategy in the use of packet loss significantly more than the number of adaptive transmission strategy. Through the comparative analysis, can explain the transmission of streaming media transmission strategies can provide better quality of service.

\section{Acknowledgement}

This work is supported by Plan Project of Youth Foundation of jiamusi University (Lq2013-032) and College students' innovative entrepreneurial training project (2014xj05)

\section{References}

[1] Hui Zhang, Chengliang Wang. Research on adaptive video streaming technology. Computer engineering and design, 2004, 2: 226 229

[2] Bing Zhou,Quanqi Bi .A MPEG stream transmission strategy of adaptive network bandwidth. Computer engineering, 2004,2: 226 229

[3] Guidong Tao, Zhangjun Zhang. Research on RTP protocol in H.264 video stream transmission based on QoS guarantee. Journal of The Academy of Armored Forces Engineering,2006,10: $58 \sim 60$

[4] Yunfei Lu, Xinggang Wang. A simulation of streaming media congestion control mechanism and performance of new. computer project, 2005,2:35 37

[5] Shining Li, Meng Wang. Long distance TCP congestion control under high bandwidth. Radio communication technology, 2006,l: 47 49

[6] Xiaoping Yang, Shuai Shi. An improved TCP congestion control algorithm.Journal of Jilin University, 2006,5: $433 \sim 435$ 